\title{
Les nations sont-elles de la compétence des
} linguistes?

Are nations a matter of linguist's competence?

Patrick Sériot

\section{OpenEdition}

\section{Journals}

Édition électronique

URL : http://journals.openedition.org//bl/438

DOI : $10.4000 / \mathrm{lbl} .438$

ISSN : $2727-9383$

Éditeur

Université de Bretagne Occidentale - UBO

\section{Édition imprimée}

Date de publication : 1 mars 2016

Pagination : 135-146

ISBN : 979-10-92331-24-0

ISSN : $1270-2412$

\section{Référence électronique}

Patrick Sériot, «Les nations sont-elles de la compétence des linguistes ? », La Bretagne Linguistique [En ligne], 20 | 2016, mis en ligne le 01 mai 2020, consulté le 01 octobre 2020. URL : http:// journals.openedition.org/lbl/438; DOl : https://doi.org/10.4000//bl.438

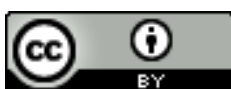

La Bretagne Linguistique est mise à disposition selon les termes de la Licence Creative Commons Attribution 4.0 International. 


\title{
Les nations sont-elles de la compétence des linguistes ? ${ }^{1}$
}

\begin{abstract}
L
a comparaison est un processus de connaissance utile, dans la mesure où un éclairage croisé peut faire apparaître des phénomènes qui seraient invisibles en éclairage direct. Ainsi en va-t-il de l'utilisation à première vue incongrue du discours sur la langue en Europe orientale pour rendre compte d'une situation linguistique éloignée, celle de la Bretagne. Mais la distance dans l'espace peut se réduire à une proximité typologique. Ainsi, parmi les termes rendant compte des entités collectives, il en est un qui est à la source de malentendus constants entre l'Est et l'Ouest de l'Europe : celui de nation. L'exemple suivant nous servira d'illustration introductive.

En 1983 paraissait un livre russe publié à Moscou en traduction française : La population du monde, de Salomon Brouk, donnant une description de chaque pays du monde d'un point de vue dit «ethno-démographique ». Quelques mois plus tard, dans L'Humanité du 29 février 1984 Georges Marchais écrivait une lettre ouverte au Comité central du PCUS, dans laquelle il exprime sa «très vive irritation » causée par ce livre. Il disait que l'auteur, "sous couvert de classification ethnographique [...] prétend diviser la population de notre pays entre, d'une part, ceux qu'il
\end{abstract}

* Université de Lausanne.

1. Cet article reprend certains éléments d'un texte plus ancien : cf. Patrick SÉRIOT, "Ethnos et Demos : la construction discursive de l'identité collective », Langages et Société (Paris, MSH), n 79, 1997, p. 39-52. 
nomme 'les Français', qui seraient, d'après lui, '44 millions, soit 82,5\% de toute la population', et d'autre part [...] les Alsaciens, les Flamands, les Bretons, les Basques, les Catalans, les Corses, les Juifs, les Arméniens, les Tziganes et 'autres'». Georges Marchais citait également un passage du livre où il est dit, par exemple, que « les Alsaciens-Lorrains s'apparentent aux Allemands ». L'argument essentiel de l'article est que

« ces allégations [sont] odieuses et ridicules. [...] Pour nous, comme pour tous les citoyens de notre pays, est français tout homme, toute femme, de nationalité française. La France n'est pas un État multinational : c'est un pays, une nation, un peuple, fruits d'une déjà longue histoire. Toute tentative - opérant sur des critères hasardeux dont la frontière avec le racisme est imprécise -, visant à définir comme non 'purement' français tels ou tels membres de la Communauté française, est une offense à la conscience nationale. Personne, ici, ne peut l'accepter, et notre Parti moins que tout autre ».

Cette réaction, me semble-t-il, repose sur une différence d'approche du problème de la nation, qui, lui-même, révèle un désaccord profond d'ordre politique et historique.

Ainsi, pour pouvoir comparer les problèmes des minorités nationales en Europe de l'Ouest et de l'Est, avant même d'essayer de trouver si les situations sont comparables, il faut interroger la notion même de comparaison et de comparabilité, et forger des instruments de comparaison. En d'autres termes, il nous faudra trouver un cadre de " commensurabilité ». Ce n'est qu'après que nous pourrons, par exemple, comparer la situation des Russes des États baltes avec celle des Français d'Algérie en 1962, ou la Moldavie avec le Pays Basque. Ce n'est qu'alors qu'on pourra décider si cela fait sens de parler de « décolonisation » à propos de l'Union soviétique se retirant des États baltes, ou si utiliser le travail qui en Europe de l'Ouest a été fait sur la Corse ou le Pays de Galles peut apporter quelque lumière sur les problèmes du Kazakhstan ou de l'Ukraine occidentale. Avant tout j'aimerais montrer combien le poids des traditions historiques et idéologiques joue un rôle dans la façon dont sont traités les problèmes des minorités nationales, et que les discours construisent des catégories qui sont ensuite pensées comme étant naturelles.

\section{La notion de « nation »}

Il y a des domaines d'investigation scientifique qui sont courants en Russie et plus rares en France. Ainsi, par exemple, le thème du " caractère national dans la langue » est sérieusement étudié par les linguistes en 
Russie $^{2}$, alors que ce même thème aurait peu de chances de trouver sa place dans des publications linguistiques universitaires en France ${ }^{3}$. Le simple fait qu'il soit étudié signifie au moins que l'existence même du caractère national d'une langue est considérée comme assurée en Russie mais plus difficilement en France, au moins en ce siècle finissant.

Pour expliquer cette différence d'approche il vaut la peine de rappeler que la notion de "nation » a une histoire différente dans les différents pays européens. Dans la France de l'Ancien Régime la langue française n'était aucunement une langue "nationale ", mais la langue nécessaire à l'administration et à l'élite intellectuelle ${ }^{4}$. La Révolution, en ce domaine, a apporté un brusque changement de point de vue : le triomphe de la langue française était le triomphe de la Nation et de la Raison. À la même époque, au contraire, en Allemagne 5 , c'est la communauté de langue qui servait à définir la nation et qui sera la base pour la revendication d'un État national unifié. La Nation française est un projet politique, né dans de violentes luttes politiques et sociales. La Nation allemande, au contraire, est apparue d'abord dans les travaux des intellectuels romantiques, comme une donnée éternelle, reposant sur une communauté de langue et de culture. Pour ces derniers la langue était l'essence de la nation, alors que pour les révolutionnaires français elle était un moyen pour parvenir à l'unité nationale. Aussi pouvons-nous, de façon simplificatrice, opposer deux définitions du mot « nation » au XIX ${ }^{e}$ siècle. En France, dans l'idéologie jacobine ${ }^{6}$, le peuple souverain proclame l'existence de la nation, une et indivisible. C'est l'État, c'est-à-dire une entité politique, qui donne naissance à la Nation, laquelle se pose alors comme « corps politique », discrète métaphore biologique. Dans la conception allemande romantique, au contraire, la Nation précède l'État ${ }^{7}$. Le «Volk» est une unité par essence, construite sur une

2. Cf. les travaux de Ju.N. Karaulov sur la «personnalité linguistique » des Russes ou le « caractère national russe dans la langue russe ».

3. Certes, il conviendrait de nuancer. Le Que-Sais-je de R. Breton sur les ethnies mériterait un examen approfondi (Roland BrETON, Les ethnies, Paris, PUF, Que Sais-je ?, 1981).

4. Du moins s'agit-il ici de la théorie implicite de la langue qu'on trouve dans l'Édit de Villers-Côtteret. Pour la vision de la langue qu'on trouve chez les Encyclopédistes, cf. Pierre ACHARD, « Mise en ordre de la langue de raison : l'État et le français », dans M.-P. Gruenais (éd.), États de langue, Paris, Fayard (Fondation Diderot), 1986, p. 4984.

5. Du moins pour Herder, dans un rapport paradoxal entre une adhésion à la philosophie des Lumières et un rejet du politique.

6. Précisons qu'il ne s'agit pas ici d'opposer des «traditions nationales ». La France du $\mathrm{XIX}^{\mathrm{e}}$ siècle a été aussi une source de la pensée contre-révolutionnaire et conservatrice des plus raffinées sur le plan intellectuel (cf. Joseph de Maistre, Louis de Bonald).

7. Précisons toutefois que ceci ne devient pleinement vrai qu'une fois que les armées 
communauté de langage et de culture. Dans la conception romantique, au commencement était la langue et la culture, alors que dans la conception contractualiste des Lumières et de la Révolution, la langue est le moyen de l'unification politique. En fait, il semble que l'idée allemand de «Kultur » soit liée à des pratiques culturelles traditionnelles, voire paysannes, alors que l'idée française de «civilisation $\|^{8}$ est plutôt liée à la ville et à des valeurs « bourgeoises », qui doivent être étendues au territoire national tout entier, au détriment de la culture paysanne (les dialectes locaux, modes de vie traditionnels, etc.). L'idée romantique allemande de nation est un système organique dans lequel la langue est porteuse d'une « culture nationale » et est liée au " peuple » d'une façon irréversible ${ }^{9}$. La conséquence en est que dans la conception romantique le peuple a déjà une langue, alors que dans la conception contractualiste la langue «commune» doit être imposée à la population entière de la nation, même, et surtout, à cette partie de la population qui ne la connaît pas. Et le fait que cette imposition n'était pas présentée comme celle de quelque chose d'étranger mais comme un raffinement de ce que les gens (le « peuple ») étaient supposés savoir déjà ne change rien à l'affaire.

Je pense que cette différence d'approche peut être résumée en termes d'ethnos et demos, c'est-à-dire dans l'opposition entre le sens romantique du mot «peuple» d'un côté, et de l'autre son sens social. La définition jacobine française de la nation est un jus soli (le droit du sol), la définition romantique allemande est un jus sanguinis (le droit du sang). L'opposition entre G. Marchais et S. Brouk repose précisément sur cette opposition entre demos et ethnos, sur une controverse implicite entre le fondement politique et le fondement ethnique de la nation, à ceci près que toute cette controverse repose sur une méconnaissance.

françaises sont passées en ayant en quelque sorte forcé toute l'Europe à faire entrer le peuple dans le jeu politique. Dans un sens il était impossible de faire la guerre aux Français sans mobiliser le peuple en lui reconnaissant des droits politiques. Mais du coup on mettait en évidence en le posant le cadre relatif des nations au pluriel, cadre que les Français présupposaient et étendaient sans précaution à ce qui était après tout l'horizon politique de l'époque.

8. Sur l'opposition entre « culture » et « civilisation », cf. A. TOYnBeE ; O. SPEngler ; Philippe Beneton, Histoire de mots : culture et civilisation, Paris, Presses de la Fondation nationale des sciences politiques, 1975 ; Alain DE BENOIST, «Culture / civilisation », Nouvelle École, 25-26, hiver 1974-75, p. 85-109.

9. Sur ce point, cf. Daniel BagGioni, « Préhistoire de la glottopolitique dans la linguistique européenne, de Herder au Cercle linguistique de Prague », Langages, $n^{\circ}$ 83, 1986, p. 35-51. 
Dans le mouvement révolutionnaire du début du $\mathrm{XX}^{\mathrm{e}}$ siècle on peut observer une opposition similaire, mais cette fois entre les marxistes d'Europe occidentale, pour qui l'appartenance de classe est le critère principal qui détermine un individu, et les marxistes d'Europe centrale et orientale (autrichiens et russes), pour qui l'appartenance nationale devait aussi être prise en compte. Il n'est pas indifférent qu'une polémique se soit engagée entre ces derniers : les « Austro-marxistes ${ }^{10}$ définissaient la nation sans égard au territoire (en tant que choix conscient et volontaire accompli par chacun à l'acquisition de la majorité), alors que pour les Bolchéviks la nation était « une communauté stable, historiquement constituée, de langue, de territoire, de vie économique et de tournure psychique, s'exprimant dans une communauté de culture ${ }^{11} \gg$. Une conséquence de cette différence est que, par exemple, dans le programme national austro-marxiste les Juifs avaient le statut de nation, alors que pour Staline les Juifs ne constituaient pas une nation ${ }^{12}$.

On peut expliquer les fluctuations de la politique nationale de l'État soviétique dans les années 20 et 30 par l'existence de deux façons de concevoir la nation. En gros, Lénine ne croyait pas à l'existence substantielle des nations, mais voulait utiliser les représentations qui s'en réclamaient comme un moyen tactique temporaire pour canaliser les mécontents locaux contre le régime tsariste, l'objectif final étant de fondre les nations (au sens de ethnos) en une nation (au sens de demos) à construire, alors que Staline insistait (du moins dans son article de 1913 et, plus tard, dans les années 30) sur le caractère " stable » de la nation, c'est-à-dire un ethnos.

Je pense que l'état actuel des problèmes nationaux en Europe de l'Est et particulièrement dans l'ex-Union soviétique peut partiellement être étudié, sinon expliqué, grâce à l'histoire des conceptions conflictuelles et

10. Cf. Otto BAuer, Die Nationalitätenfrage und die Sozialdemokratie, Wien, 1924 (trad. fr. : La question des nationalités et la social-démocratie, Paris, Arcantèle, 1987).

11. Cf. Joseph Staline, « Marksizm i nacional'nyj vopros », dans I. Stalin, Sochinenija, t. 2, Moscou, [1913] 1953 [Le marxisme et la question nationale].

12. Cf. Hélène CARrère D’EnCAusse, Le grand défi : Bolcheviks et nations, 1917-1930, Paris, Flammarion, 1987. De même, pour Staline (op. cit., [1913] 1953, p. 294) : « Il n'est pas de nation qui parle à la fois plusieurs langues », ce qui semble exclure que la Suisse constitue une nation, alors que pour le linguiste Albert DAUZAT (L'Europe linguistique, Paris, Payot, 1953 (1re éd. 1940), p. 16), « un État bilingue ou trilingue peut former une nation animée d'un sentiment patriotique commun à ses différents éléments. L'exemple le plus frappant est celui de la Suisse...». Cf. le même raisonnement chez Ernest RENAN, «Qu'est-ce qu'une nation?», Association scientifique de France, Bulletin hebdomadaire, 26 mars 1882 (repris dans Qu'est-ce qu'une nation?, Paris, Press-Pocket, coll. Agora, 1992). 
incompatibles de la nation. On peut remarquer, par exemple, que les idéologues principaux de la politique nationale de l'époque dite de la « stagnation » [zastoj], M. Bromlej et Ju. Guboglo, ont élaboré une théorie de l'« ethnos » (c'est-à-dire à la façon romantique) qui s'est avérée être la base d'une politique de destruction des autonomies locales à l'intérieur de l'État unique d'un peuple unique (c'est-à-dire à la façon contractualiste). D'autre part, ce conflit constant entre demos et ethnos peut être utilisé comme une clé pour expliquer certaines différences entre les mouvements nationaux minoritaires d'Europe de l'Ouest et de l'Est à l'heure actuelle.

\section{L'ordre du discours}

Les faits et les événements historiques sont empiriquement toujours différents, toujours nouveaux. Mais un aspect de leur « commensurabilité » est le discours qui est tenu à leur sujet. Un événement historique ne prend son sens, c'est-à-dire ne peut être traité, ou avoir des conséquences pratiques, que dans les mots qui sont utilisés pour en parler. Cet ensemble de mots peut être séparé en différents sous-ensembles, parfois incompatibles entre eux, qui forment différents discours. L'organisation matérielle de ces discours (organisation rhétorique, lexicale et même grammaticale) peut être étudiée et comparée. Tout particulièrement l'usage des noms, en tant qu'ils construisent des catégories conceptuelles, doit être étudié avec $\operatorname{soin}^{13}$.

La nation n'est pas un objet naturel, mais une catégorie, qui existe avant tout dans le nom qu'une communauté se donne à elle-même ou que d'autres lui donnent de l'extérieur (cf. J.-P. Sartre et ses réflexions sur la question juive) ${ }^{14}$. Un ethnonyme n'est pas le reflet direct d'une « chose », mais un objet de discours. Dans cette perspective la lutte pour le nom a exactement la même importance à l'Ouest qu'à l'Est, car l'enjeu de la nomination est l'expression de l'être. Par exemple, le slogan du Front Populaire en Moldavie après 1989 était : « Nous ne sommes pas des Moldaves, nous sommes des Roumains », ou plus précisément une citation de M. Eminescu : «Suntem români si punctum» (Nous sommes Roumains

13. Cf. P. SÉRIOT, «Faut-il que les langues aient un nom ? Le cas du macédonien », dans A. Tabouret-Keller (éd.), Le nom des langues. Les enjeux de la nomination des langues, Louvain, Peeters, 1997, p. 167-190..

14. Si en général une nation a un nom, toute communauté nommée n'est pas nécessairement une nation. L'exemple de l'Israel biblique montre d'ailleurs qu'une nation peut être définie pour elle-même par le fait de ne pas avoir de nom (ou plus exactement que « notre nom » n'est pas un nom de nation, que nous ne sommes pas une nation). 
et point), tout comme dans le Roussillon on trouve couramment peint sur les murs le slogan «Sem pa francès, sem catalá » (Je ne suis pas Français, je suis Catalan). Le nom est un objet symbolique qui donne existence à un groupe, il crée une discontinuité dans une continuité. Il transforme les groupes en objets dénombrables. Ainsi S. Brouk oppose deux nations dans la région de Tchernovsty (en Ukraine) : les Moldaves et les Roumains (sans d'ailleurs donner de critères de distinction : celle-ci semble être une donnée d'évidence). Fabriquer du discontinu à l'intérieur d'un continu a de nombreuses conséquences pratiques et matérielles. Par exemple, le moldave et le roumain sont-ils des langues différentes ou une seule et même langue ? D'un strict point de vue descriptif, cette question n'a pas de sens. Mais d'un point de vue politique, elle a des conséquences directes. La tâche des académiciens soviétiques officiels après la deuxième guerre mondiale était de démontrer que ces langues étaient différentes, ce qui permettait au gouvernement central de changer l'alphabet de la Bessarabie du latin au cyrillique et de ne faire des néologismes que sur la base du russe ${ }^{15}$.

Discuter le nom d'une langue relève du même type d'opérations que discuter le nom d'une nation. Par exemple, la nation macédonienne existet-elle ? Du point de vue yougoslave officiel (du moins à l'époque de Tito), le macédonien est une langue, ce qui fait que la Macédoine a reçu le statut de République fédérée en Yougoslavie. Du point de vue bulgare, en revanche, le macédonien n'est pas une langue, mais une variante locale du bulgare, ce qui a permis au gouvernement bulgare à l'époque communiste d'avoir des revendications territoriales sur le Sud de la Yougoslavie et de ne pas accorder le statut de minorité nationale aux Macédoniens de Bulgarie.

Je ne propose pas ici de séparer la vérité de la fausseté : ce qui est en question n'est pas l'adéquation des mots aux choses, mais le fait que certains mots sont acceptés ou non, sont pris pour acquis ou non, pour désigner des communautés, c'est-à-dire pour regrouper ou pour séparer. C'est le nom qui fait la frontière. Un exemple de cette affirmation peut être trouvé dans le cas des "Polesie». Ces gens vivent en Biélorussie, en Ukraine et en Pologne. Jusqu'à la fin des années 80, ils n'avaient pas de nom officiellement reconnu. Dans la région polonaise de Bialystok, le journal biélorusse local Niva publie des poèmes dans le « dialecte polesie du biélorusse ». Mais le 14 avril 1988 est apparue une nouvelle organisa-

15. Les événements contemporains de la fin de la perestrojka en URSS ont inversé les données du problème, avec le passage de l'alphabet cyrillique à l'alphabet latin en Moldavie ex-soviétique, sans que d'ailleurs soit réglé le problème du nom de la langue qui y était parlée : « moldave » ou « roumain »? 
tion culturelle : Polesie, revendiquant la résurrection de la culture, de la langue et du territoire polesie, sans tenir compte des frontières d'État entre l'Ukraine et la Biélorussie (cf. Sheljagovich ${ }^{16}$ ). Une fois encore, c'est sur cette base qu'est possible une comparaison est/ouest : le conflit discursif le plus important dans la presse française pendant la guerre d'Algérie était de décider si l'Algérie était ou n'était pas la France (cf. Maldidier) ${ }^{17}$.

Donner des noms est une façon de cristalliser de nouvelles oppositions de groupes. Ainsi dans la Russie post-perestrojkiste dans le discours des mouvements russes ultra-nationalistes est apparue l'opposition discursive mondialisty, atlantisty vs patrioty, evrazijcy [eurasiens], traçant ainsi une nouvelle partition entre « eux » et « nous ».

Une façon complémentaire de fabriquer une identité est d'amener un adversaire, un " autre » à l'existence en lui donnant un nom. Il y a en ce moment plusieurs façons de se nommer soi-même pour les mouvements nationalistes russes, selon le type d'opposition dans lesquels ils se placent. Pour l'écrivain Rasputin il y a les Slaves et les non-Slaves. La conséquence est qu'un Polonais ou un Serbe sera considéré par lui comme «nous ", alors qu'un Allemand ou un Arménien sera «eux». Pour d'autres, par exemple L. Gumilev, au contraire, la ligne de rupture passe entre les « Eurasiens » et les « Occidentaux ». En ce cas, un Polonais ou un Serbe feront partie de " eux » tout comme un Allemand, mais un Tatar ou un Kalmouk seront « nous ». Une conséquence de la définition « ethnique» de la nation est une nouvelle partition entre les noms des Russes. Un « droit du sang » nécessite deux noms : les russkie sont tous les membres de la nation russe, où qu'ils vivent (par exemple dans la « République de Trans-Nistrie »), alors que rossijane est le nom des habitants du territoire de la Russie, ce qui signifie qu'un Tatar vivant sur les rives de la Volga est dit être un rossijanin par ceux qui donnent au territoire de l'ex-RSFSR le statut d'un nouvel État indépendant. Mais un Tatar nationaliste, bien sûr, ne peut pas se désigner lui-même comme rossijanin s'il milite pour l'indépendance du Tatarstan. La longue polémique du parlement russe du mois d'avril 1992 sur le nom du pays («Russie» ou «Fédération russe ») reflète l'importance de

16. M. Sheljagovich, «Praktika osparivaet teoriju» [La pratique met la théorie en question], s.d.

17. Le problème de la nomination de l'Algérie avait des implications légales précises : la constitution stipule que la nation est indivisible, et dire que l'Algérie c'est la France c'est interdire un référendum sans révision de la constitution. Le nom des Polesie doit tôt ou tard poser des problèmes de nature semblable. D. MALDIDIER, « Le discours politique de la guerre d'Algérie : approche synchronique et diachronique », Langages, 23, 1971. 
la fabrication discursive de l'identité. Rappelons-nous, enfin, la lutte des Moldaves pour changer leur alphabet ou les débats sur le changement de nom de la ville de Léningrad, et il deviendra clair que, d'abord, l'identité symbolique a des conséquences matérielles, qu'ensuite les frontières et les limites ne sont pas des objets naturels, et qu'enfin comparer des formations discursives est une façon de trouver une commune mesure pour étudier les mouvements nationaux en Europe de l'Est et de l'Ouest et expliquer leurs différences de comportement.

\section{Un discours majoritaire en position de minorité}

Prenons maintenant pour exemple le discours nationaliste des minorités russes dans les nouveaux États issus de l'ex-URSS. Une particularité du discours nationaliste russe est que l'appartenance nationale des Russes doit être justifiée selon une hiérarchie de niveaux. Les différentes variétés du discours nationaliste russe insistent sur la lutte contre l'internationalisation, contre le nivellement des valeurs à l'échelle mondiale (ce qui voudrait dire accepter les standards occidentaux) ou contre une destruction cosmopolite de la Russie (ce qui désigne les Juifs). Ce qui est en jeu est la possibilité de « conserver sa culture ». Mais, d'un autre côté, il est nécessaire d'insister sur la communauté naturelle de tous les peuples de l'exURSS (la tendance « eurasienne »), ou de tous les peuples de la Fédération de Russie. Si, auparavant, pendant l'ère brejnévienne, on disait que ces peuples avaient une «tendance » à se rapprocher ou même à fondre leur langue dans la langue russe, à l'heure actuelle on insiste plutôt sur la proximité naturelle (même si historiquement déterminée) de ces peuples avec la culture russe. C'est ainsi qu'est donnée une base naturelle, voire nécessaire pour justifier l'existence de la Fédération de Russie en tant que telle.

La façon dont les minorités russes parlent d'elles-mêmes dans les nouveaux États issus de l'ancienne URSS est fondamentalement unanimiste, c'est-à-dire qu'elle s'appuie essentiellement sur une définition ethnique de la nation. En Écosse, en Corse, dans la partie occitane du Sud de la France, les mouvements nationalistes ont de fortes revendications économiques et sociales. Ici ethnos et demos sont plus ou moins liés. La conscience nationale est apparue en même temps que la conscience du statut économique inférieur des régions périphériques par rapport à un « centre » économiquement plus développé. C'est la motivation de slogans tels que volem viure al pais (Nous voulons vivre dans notre pays) qui furent soutenus par les organisations d'extrême-gauche en France dans les années 1968-1980. En revanche, le discours des minorités nationales russes en dehors de la Fédé- 
ration de Russie, pour autant qu'on peut en juger sur la base de journaux et de tracts, repose essentiellement sur une conception de la nation comme « ethnos ». Une assertion mainte fois répétée par les Russes des États baltes, par exemple, est qu'ils sont en danger, non pas en tant que catégorie sociale, mais en tant que groupe ethnique. Ils considèrent comme une atteinte aux « Droits de l'homme » le fait d'avoir à apprendre la langue locale pour avoir accès à une activité professionnelle publique. Ils font régulièrement appel au Gouvernement russe pour demander sa «protection ». La fabrication discursive de l'identité de leurs adversaires repose sur le fait que ces derniers « ne les aiment pas », et non sur des revendications démocratiques, qui pourraient mener à discuter leur position comme étant une pure appartenance ethnique et non comme une certaine position sociale dans la république. Le journal nationaliste Zavtra expose une argumentation forte, tendant à prouver que la perte des Etats baltes est la première étape d'un complot contre la nation russe, fomenté à la fois par les Atlantisty (ou « Occidentalistes ») et les Juifs. Le discours nationaliste des Baltes semble plus diversifié. Il semble que le discours estonien prend en considération la position économique des ouvriers russes dans les usines d'armement de la région de Narva. En 1992, le discours du Président Landbergis, d'autre part, construit une autre catégorie d'étrangers (un autre " eux ») : les Polonais, à l'intérieur de la Lituanie. En janvier 1992 il déclarait à la télévision polonaise que les Polonais avaient joué un rôle «passif » pendant le putsch d'août 1991, justifiant ainsi qu'aucune représentation parlementaire n'ait été donnée en Lituanie aux Polonais en tant que tels.

Une fois encore, on ne propose pas ici une échelle de valeurs, mais on essaye de donner les moyens d'une comparaison est/ouest en ce qui concerne les discours des minorités : les frontières de l'identité collective sont construites dans et par des discours. On peut alors proposer une échelle de classification avec une opposition bi-polaire entre deux points extrêmes, depuis un fort unanimisme (sur la base de la langue, de l'ethnicité ou de la religion, c'est-à-dire une hypertrophie du principe de l'ethnos) jusqu'à une large prise en compte de la division sociale (le principe du demos). Mais cette opposition n'est ni ontologique ni culturelle : si dans les deux cas le mot peuple désigne symboliquement le même référent, il le construit dans un espace de propriétés distinct. On peut penser que toute conception de la nation possède les deux dimensions, mais que l'une tend à être présupposée alors que l'autre est posée. Il reste à étudier attentivement pourquoi l'universalisme centré qui caractérise l'articulation entre ethnos et demos en France est si opaque vu de l'Est (mais aussi peutêtre vu d'ailleurs) et inversement pourquoi la partition entre citoyenneté 
et nationalité, composante intrinsèque du droit soviétique et de nombreux États d'Europe orientale encore actuellement est si étrange vue de France.

On donnera alors en conclusion quatre propositions :

- laissez les gens parler la langue qu'il leur plaît ;

- une langue d'État est une chose pragmatiquement utile ;

- le bilinguisme est un projet réaliste ;

- ce qu'on dit dans une langue est plus important que la langue dans laquelle on le dit.

\section{Bibliographie récapitulative}

ACHARD Pierre, «Mise en ordre de la langue de raison : l'État et le français », dans M.-P. Gruenais (éd.), États de langue, Paris, Fayard (Fondation Diderot), 1986, p. 49-84.

BAGGIONI Daniel, "Préhistoire de la glottopolitique dans la linguistique européenne, de Herder au Cercle linguistique de Prague », Langages, $\mathrm{n}^{\circ} 83,1986$, p. $35-51$.

BAUER Otto, Die Nationalitätenfrage und die Sozialdemokratie, Wien, 1924 (trad. fr. : La question des nationalités et la social-démocratie, Paris, Arcantèle, 1987).

BENETON Philippe, Histoire de mots : culture et civilisation, Paris, Presses de la Fondation nationale des sciences politiques, 1975.

BRETON Roland, Les ethnies, Paris, PUF, Que Sais-je ?, 1981.

CARRÈre D'ENCAUSSE Hélène, Le grand défi : Bolcheviks et nations, 19171930, Paris, Flammarion, 1987.

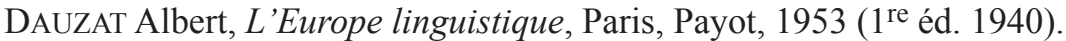

DE BENOIST Alain, "Culture / civilisation », Nouvelle École, 25-26, hiver 1974-75, p. 85-109.

MALDIDIER D., «Le discours politique de la guerre d'Algérie : approche synchronique et diachronique », Langages, 23, 1971.

RENAN Ernest, "Qu'est-ce qu'une nation?», Association scientifique de France, Bulletin hebdomadaire, 26 mars 1882 (repris dans Qu'est-ce qu'une nation?, Paris, Press-Pocket, coll. Agora, 1992).

SÉRIOT Patrick, «Faut-il que les langues aient un nom ? Le cas du macédonien », dans A. Tabouret-Keller (éd.), Le nom des langues. Les enjeux de la nomination des langues, Louvain, Peeters, 1997, p. 167-190.

SÉRIOT Patrick, «Ethnos et Demos : la construction discursive de l'identité collective », Langages et Société (Paris, MSH), 1997, nº 79, p. 39-52. 
SÉRIOT Patrick, Les langues ne sont pas des choses. Discours sur la langue et souffrance identitaire en Europe centrale et orientale, Paris, Petra, 2010. ShelJAGOVICH M., « Praktika osparivaet teoriju » [La pratique met la théorie en question], s.d.

STALIN Joseph, « Marksizm i nacional'nyj vopros », dans I. Stalin, Sochinenija, t. 2, Moscou, [1913] 1953 [Le marxisme et la question nationale]. 